\title{
Esophageal Ulcer, CTCAE
}

National Cancer Institute

\section{Source}

National Cancer Institute. Esophageal Ulcer, CT CAE. NCI Thesaurus. Code C143460.

A disorder characterized by a circumscribed, erosive lesion on the mucosal surface of the esophageal wall. 